\section{Gynäkologisch- Rundschau}

\author{
Abschiedsvorlesung 180 \\ Affekte 109 \\ Akzeptanz 242 \\ Amenorrhö 47 \\ Angstneigung 232 \\ Appendixendometriose 21 \\ Arbeit und Schwangerschaft 211 \\ Artifizielle Insemination, Spender 10 \\ Ausbildung 180
}

Bauchwanddefekte 216

Bisphosphonate 64

Borrelia burgdorferi 143

Brustkrebs 101

Carcinoma in situ 193

Chemische Peritonitis 146

Chemoprävention 105

Clodronat 64

Dermoide Ovarialzyste 146

Deszensus 242

Deziduale Umwandlung 21

Edinburgh Postnatal Depression Scale 232

Eklampsie 151

Endometriumkarzinom 73, 93, 96, 105

Epidermaler Wachstumsfaktor 238

Fertilität 41

Fetaler Folgezustand 158

Follikuläre lymphatische Hyperplasie 143

Fruchtwasser 16

Frühgeburt 131, 188

Ganzheitliche Geburtshilfe 188

Gastroschisis 216

Geburtsbelastung 232

Geburtsdauer 158

Geburtshilfliches Management 131

Geschichte der Geburtshilfe 25

Gesundheitsökonomie 196

Gossypol 50

Gravidin 16

Gynäkologische Malignome 109

Hausgeburtshilfe 196

HELLP-Syndrom in der 32. SSW 21

Historischer Rückblick 41

Hormonale Methoden 50

Hormonersatztherapie 89

Hypergonadotroper Hypogonadismus 10

Hysteroskopische Endometriumablation,

Techniken 119

- Endometriumresektion, Indikationen 119
Implantation 44

Intrauterine Wachstumsretardierung 238

Intrazytoplasmatische Spermieninjektion 10

In-vitro-Fertilisation 10

Kaiserschnitt 232

Kapazitation 44

Kardiovaskuläre Protektion 96

Klinikgrösse 131

Knochendichte 96

Knochenmetastasen 64

Komplikationen 3

Kryokonservierung 10

Laparoskopie 146

Lebensqualität 109

Lyme-Krankheit 143

Lymphadenektomie 85

Magnetresonanztomographie 193

Mammakarzinom 64, 73, 81

Medizingeschichte 25

Menstruation 44

Menstruationszyklus 151

Metaanalyse 81, 101

Mikrochirurgische epididymale Spermienaspiration 10

Mikrokarzinom 193

Mutterschaftsurlaub 211

Nachbeobachtung 216

Nebenwirkungen adjuvanter Hormontherapien 96

Niederlande 196

Omphalozele 216

Operative Gynäkologie 180

Orale Kontrazeptiva 101, 105

Österreich 196

Östrogenersatztherapie 93

Ovarialinsuffizienz 47

Ovarialkarzinom 73, 89, 105

Ovulation 44

Partus 16

Pessar 242

Phospholipase- $\mathrm{A}_{2}$-Inhibitor 16

Pille für den Mann 50

Polymerasekettenreaktion 143

Postoperative Bestrahlung 3

Postpartum-Depression 232

Prädiktion 222

Präeklampsie 151, 222

Prämenopause 109

Pränatale Diagnostik 216
Primärbestrahlung 3

Profane Geschichte 25

Prophylaxe 64

Prostaglandine 188

Pseudolymphom 143

Radikale abdominale Hysterektomie 85

Radikaloperation 3

Risikoreduktion 105

Roma-Geburtsrad 158

-, Zufriedenheit 158

Schädellagegeburten 158

Schnittentbindung 131

Schwangerenvorsorge 196

Schwangerschaft 85

Sectio 188

Serum 222

Sicherheit 242

Sterilität 41

Stickstoffmonoxid 44

Stressinkontinenz 242

Tamoxifen 81

Testikuläre Spermienextraktion 10

Therapieresistente Blutungsstörungen 119

Tokographie 151

Tokolyse 188

Trauer 109

Trophoblast 222

Tumorvolumen 193

Überlebensrate, Endometriumkarzinom 93

Überwachung des fetalen Wachstums 238

Ultraschall 216

Universitäts-Frauenklinik Rostock, Geschichte 151

Vaginale Uterusexstirpation 180

Verlust 109

Vorzeitiger Mutterschutz 211

Vulvaerkrankungen 143

Vulväre Hauterkrankungen 143

Wehen 16

Wochenbett 196

Zervikale intraepitheliale Neoplasie 85

Zervixkarzinom 3, 73, 85, 89

Zyklusanomalie 47 